\title{
Dynamical $r$-matrices and Poisson-Lie symmetries in the chiral WZNW model
}

\author{
László Fehér* \\ Department of Theoretical Physics, University of Szeged \\ Tisza Lajos krt 84-86, H-6720 Szeged, Hungary \\ E-mail: 'ifeher@sol.cc.u-szeged.hu'
}

ABstract: We briefly review the possible Poisson structures on the chiral WZNW phase space and discuss the associated Poisson-Lie groupoids. Many interesting dynamical $r$ matrices appear naturally in this framework. Particular attention is paid to the special cases in which these $r$-matrices satisfy the classical dynamical Yang-Baxter equation or its Poisson-Lie variant.

\section{Introduction}

Let me start by recalling the standard classical dynamical Yang-Baxter equation (CDYBE),

$$
\left[r_{12}(\lambda), r_{23}(\lambda)\right]+H_{1}^{i} \frac{\partial}{\partial \lambda^{i}} r_{23}(\lambda)+\text { cycl. perm. }=0,
$$

where $r(\lambda) \in \mathcal{G} \otimes \mathcal{G}$ and the variable $\lambda=\lambda^{i} H_{i}$ lies in a Cartan subalgebra of a simple Lie algebra. This equation is the classical limit of the Gervais-Neveu-Felder equation

$$
R_{12}\left(\lambda+\hbar H_{3}\right) R_{13}(\lambda) R_{23}\left(\lambda+\hbar H_{1}\right)=R_{23}(\lambda) R_{13}\left(\lambda+\hbar H_{2}\right) R_{12}(\lambda) .
$$

These equations govern the classical and quantum exchange algebras of the chiral Bloch

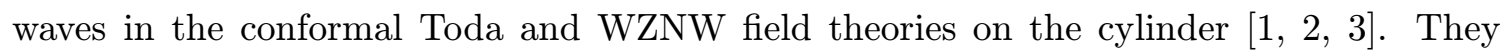
also appear in the description of the conformal blocks of the WZNW model on the torus

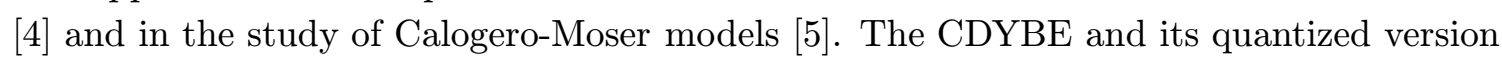
have interesting generalizations that play an important role in quantum algebra and in the theory of integrable systems [i6].

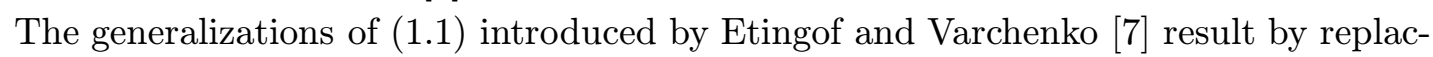
ing the Cartan subalgebra by (the dual space of) any subalgebra $\mathcal{H}$ of any Lie algebra $\mathcal{G}$. Of course, one can also consider the spectral parameter dependent variant of these

\footnotetext{
${ }^{*}$ Speaker.
} 
equations. The most important special case, related to affine Kac-Moody algebras, is when the 'dynamical variable' $\lambda$ belongs to the fixed point set of a Coxeter automorphism of a simple Lie algebra.

In the context of the classical WZNW model, the variable $\lambda$ in $\left(\begin{array}{l}1 \\ 1\end{array} \overline{1}_{1}^{\prime}\right)$ is the logarithm of the monodromy of the chiral WZNW field. Motivated by our longstanding interest in the WZNW model as well as by the intense current research activity around the CDYBE, with J. Balog and L. Palla [i8 is we have recently explored the most general Poisson structure that arises on the chiral WZNW phase space without imposing the constraint that the monodromy belongs to a maximal torus. It turned out that these Poisson structures are parametrized by solutions of a generalization of the CDYBE, which we call the G-CDYBE. We can actually find all solutions of the $G$-CDYBE as part of our analysis of the WZNW model, which is

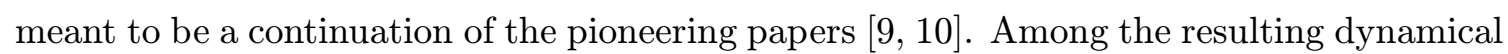
$r$-matrices there is a particularly interesting class associated with Poisson-Lie symmetries acting on the chiral WZNW phase space. When the Poisson-Lie symmetry degenerates into usual symmetry, then these $r$-matrices reduce to a canonical solution of the CDYBE on $\mathcal{G}$ in the sense of $[\bar{i}]$, which upon further Dirac reduction to suitable subalgebras of $\mathcal{G}$ (and by certain limiting procedures) reproduces many of the known $r$-matrices of the Etingof-Varchenko type.

Here I present a brief review of the main results that we obtained in [8], touching also on their further clarifications published in $\left[{ }_{1}^{1} \overline{1}_{0}^{1}, \overline{1} 2\right]$. For lack of time, I cannot deal with

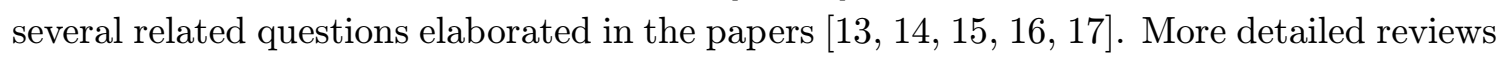
of some aspects of our work can be found in [i] $\left[\begin{array}{l}\overline{8} \\ 0\end{array}\right.$

\section{G-CDYBE from the chiral WZNW phase space}

The WZNW model [20̄] as a classical field theory on the cylinder can be defined for any (real or complex) Lie group $G$ whose Lie algebra $\mathcal{G}$ is self-dual in the sense that it is equipped with an invariant, symmetric, non-degenerate bilinear form $\langle$,$\rangle . The solution$ of the classical field equation for the $G$-valued WZNW field, which is $2 \pi$-periodic in the space variable, is given by the product of left- and right-moving chiral WZNW fields that are quasi-periodic. By restricting the 'monodromy matrix' to lie in some open submanifold $\check{G} \subseteq G$, we thus obtain the chiral WZNW phase space

$$
\mathcal{M}_{\check{G}}:=\left\{g \in C^{\infty}(\mathbf{R}, G) \mid g(x+2 \pi)=g(x) M \quad M \in \check{G}\right\} .
$$

If one wishes to induce the Poisson structure of the full WZNW model from Poisson brackets (PBs) of chiral fields varying in $\mathcal{M}_{\breve{G}}$, then the only possibility is to equip $\mathcal{M}_{\breve{G}}$ with a PB of the following form:

$$
\kappa\{g(x) \stackrel{\otimes}{,} g(y)\}_{W Z}^{r}=(g(x) \otimes g(y))\left(\frac{1}{2} C \operatorname{sign}(y-x)+r(M)\right), \quad 0<x, y<2 \pi .
$$

Here the interesting object is the 'exchange r-matrix' $r(M)=r^{a b}(M) T_{a} \otimes T_{b} \in \mathcal{G} \wedge \mathcal{G}$; $C=T_{a} \otimes T^{a}$ where $\left\{T_{a}\right\}$ and $\left\{T^{a}\right\}$ denote dual bases of $\mathcal{G},\left\langle T_{a}, T^{b}\right\rangle=\delta_{a}^{b}$. One way to 
derive this $\mathrm{PB}$ is to invert the symplectic form $\Omega_{W Z}^{\rho}$ on $\mathcal{M}_{\breve{G}}$ found by Gawedzki [ig]

$$
\frac{1}{\kappa} \Omega_{W Z}^{\rho}(g)=-\frac{1}{2} \int_{0}^{2 \pi} d x\left\langle\left(g^{-1} d g\right) \wedge\left(g^{-1} d g\right)^{\prime}\right\rangle-\frac{1}{2}\left\langle\left(g^{-1} d g\right)(0) \wedge d M M^{-1}\right\rangle+\rho(M)
$$

with some 2 -form $\rho$ on $\check{G}$. Another method that leads directly to $\left(\overline{2} \cdot \overline{2} \overline{2}_{i}\right)$ uses the requirements that $J:=\kappa g^{\prime} g^{-1}$ must be an affine Kac-Moody current with respect to which $g$ is a chiral primary field, and that the WZNW solution space must be obtained from the product of two independent chiral phase spaces by imposing first class constraints. Both methods

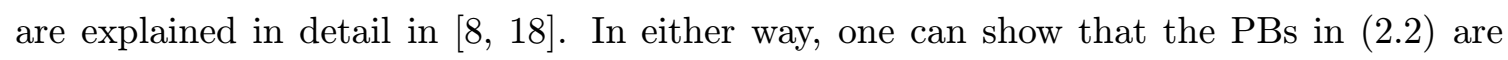
accompanied by

$$
\kappa\{g(x) \stackrel{\otimes}{,} M\}_{W Z}^{r}=(g(x) \otimes M) \Theta(M) \quad \text { and } \quad \kappa\{M \stackrel{\otimes}{,} M\}_{W Z}^{r}=(M \otimes M) \Delta(M),
$$

with

$$
\Theta(M)=r^{+}(M)-M_{2}^{-1} r^{-}(M) M_{2}, \quad \Delta(M)=\Theta(M)-M_{1}^{-1} \Theta(M) M_{1}
$$

where $r^{ \pm}:=r \pm \frac{1}{2} C, M_{1}=M \otimes 1, M_{2}=1 \otimes M$. In fact [8i], the Jacobi identity ${ }^{1}$ of the PB (2) is equivalent to the following equation:

$$
\left[r_{12}(M), r_{23}(M)\right]+T_{1}^{a}\left(\frac{1}{2} \mathcal{D}_{a}^{+}+r_{a}^{b}(M) \mathcal{D}_{b}^{-}\right) r_{23}(M)+\text { cycl. perm. }=-\frac{1}{4} \hat{f} .
$$

Here $\hat{f}:=f_{a b}{ }^{c} T^{a} \otimes T^{b} \otimes T_{c}$ with $\left[T_{a}, T_{b}\right]=f_{a b}{ }^{c} T_{c}, r_{23}=r^{a b}\left(1 \otimes T_{a} \otimes T_{b}\right)$ and $T_{1}^{a}=T^{a} \otimes 1 \otimes 1$ as usual; for any function $\psi$ on $G$ we use

$$
\mathcal{D}_{a}^{ \pm}=\mathcal{R}_{a} \pm \mathcal{L}_{a} \quad \text { with } \quad\left(\mathcal{R}_{a} \psi\right)(M):=\left.\frac{d}{d t} \psi\left(M e^{t T_{a}}\right)\right|_{t=0}, \quad\left(\mathcal{L}_{a} \psi\right)(M):=\left.\frac{d}{d t} \psi\left(e^{t T_{a}} M\right)\right|_{t=0} .
$$

We call equation (2. 6 ) the $G$-CDYBE since it is a generalization of the CYBE for an $r$-matrix depending on a $G$-valued 'dynamical variable'. The $G$-CDYBE becomes the standard modified-CYBE if $r$ is an $M$-independent constant.

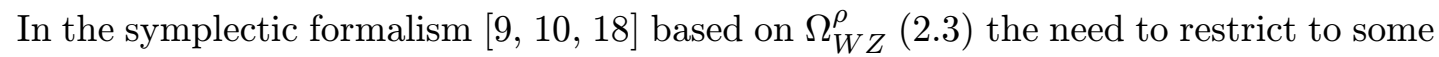

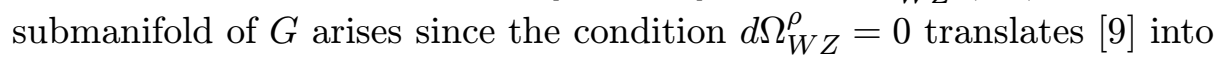

$$
d \rho=\frac{1}{2}\left\langle\left[M^{-1} d M, M^{-1} d M\right], M^{-1} d M\right\rangle,
$$

which in general does not admit a global solution on $G$, as is well known. In the alternative approach in which $\left(\mathcal{M}_{\breve{G}},\{,\}_{W Z}^{r}\right)$ is required to be a (not necessarily non-degenerate) Poisson space, the only condition is that $r: \check{G} \rightarrow \mathcal{G} \wedge \mathcal{G}$ must be a regular (smooth or holomorphic) solution of the $G$-CDYBE (2.2.6.

Note that the exchange $r$-matrix can be constant, which is the case considered mostly

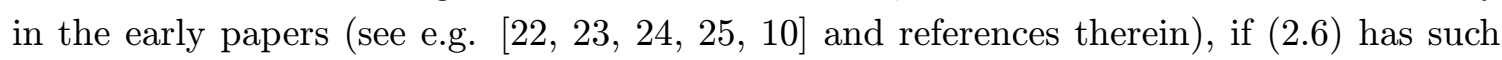
a solution. The real forms of the simple Lie algebras that admit a constant solution [20 $\left.\bar{\sigma}_{1}\right]$ include the split real form, but exclude the compact one.

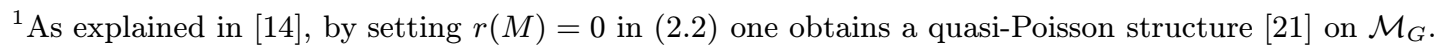




\section{The canonical $r$-matrix and its Dirac reductions}

We actually have all solutions of the $G$-CDYBE locally in a neighbourhood of $e \in G$. More precisely, we have an explicit one-to-one correspondence between the solutions $\rho$ of $(2.8)$ and the solutions $r$ of $(2.6)$ around $e \in G$. Such a correspondence was derived originally by inverting $\Omega_{W Z}^{\rho}$ [is] [1]

A particularly interesting exchange $r$-matrix arises if the PB (2.2i) permits the 'gauge action' of the group $G$ on $\mathcal{M}_{\check{G}}$, given by

$$
\mathcal{M}_{\check{G}} \times G \ni(g(x), h) \mapsto g(x) h,
$$

to operate as a classical $G$-symmetry generated by the logarithm of the monodromy matrix.

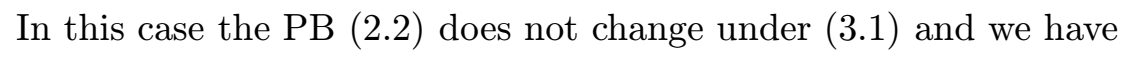

$$
\kappa\left\{g(x), m_{a}\right\}_{W Z}^{r}=g(x) T_{a}, \quad \kappa\left\{m_{a}, m_{b}\right\}_{W Z}^{r}=-f_{a b}{ }^{c} m_{c} \quad \text { for } \quad M=e^{m},
$$

where $m=\log M$ lies in a neighbourhood of zero, $\check{\mathcal{G}} \subset \mathcal{G}$, diffeomorphic to $\check{G}$ by the exponential map. In the notion of 'classical symmetry' it is understood that the elements of the symmetry group have zero PBs with everything. Let $r^{0}(m)$ denote an exchange

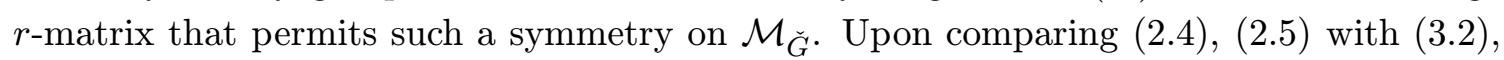

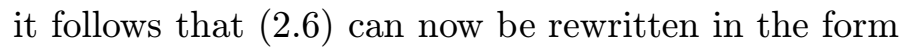

$$
\left[r_{12}^{0}(m), r_{23}^{0}(m)\right]+T_{1}^{a} \frac{\partial}{\partial m^{a}} r_{23}^{0}(m)+\text { cycl. perm. }=-\frac{1}{4} \hat{f}
$$

and it is also easy to see that $r^{0}(m)$ must be $G$-equivariant,

$$
r^{0}\left(h m h^{-1}\right)=(h \otimes h) r^{0}(m)\left(h^{-1} \otimes h^{-1}\right) \quad \forall h \in G .
$$

It is natural to search for an equivariant $r$-matrix by using the ansatz

$$
r^{0}(m)=\left\langle T_{a}, f_{0}(\operatorname{ad} m) T_{b}\right\rangle T^{a} \otimes T^{b},
$$

where $f_{0}(z)$ is assumed to be a holomorphic, odd complex function in a neighbourhood of

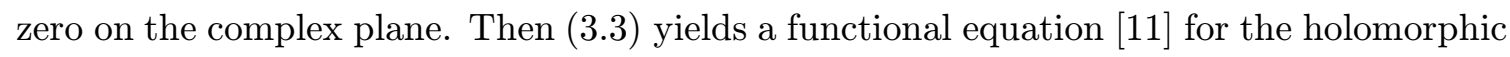
function $f_{0}$, whose unique solution is provided by

$$
f_{0}(z)=\frac{1}{2} \operatorname{coth} \frac{z}{2}-\frac{1}{z} .
$$

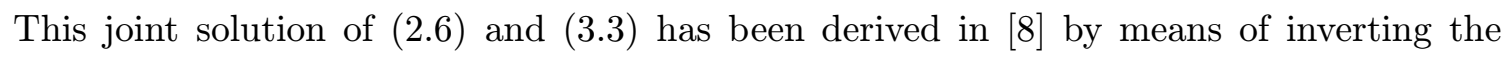
symplectic form $\left(\overline{2} \cdot \overline{3}^{i}\right)$ for the 2 -form

$$
\rho_{0}(M)=-\frac{1}{2} \int_{0}^{2 \pi} d x\left\langle d \bar{m} \wedge d e^{x \bar{m}} e^{-x \bar{m}}\right\rangle, \quad \bar{m}:=\frac{1}{2 \pi} \log M,
$$

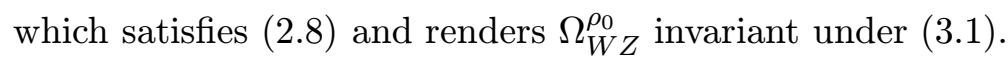




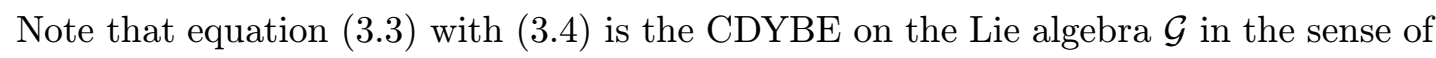
[i] (or 'modified' CDYBE on account of the non-zero right-hand side). Somewhat implicitly, the solution of the CDYBE on a simple Lie algebra given by $(\overline{3} \cdot \overline{3}),(\overline{3} \cdot \overline{6})$ is already contained in [i] $]$. It was also found in $[\overline{2} \overline{7}]$ in the context of equivariant cohomology. Its uniqueness property under the ansatz (3.5) is proven in (This uniqueness property should be compared with the description of the 'moduli space'

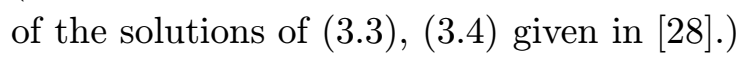

The canonical $r$-matrix described above plays a distinguished role among the solutions of the CDYBE in the Etingof-Varchenko sense. To explain this, let $\mathcal{H} \subset \mathcal{G}$ be a selfdual subalgebra (on which $\langle$,$\rangle remains non-degenerate), and consider the associated$ decomposition $\mathcal{G}=\mathcal{H} \oplus \mathcal{H}^{\perp}$. Then define $r^{*}: \check{\mathcal{H}} \rightarrow \operatorname{End}(\mathcal{G})$ by

$$
r^{*}(\lambda)(X)=f_{0}(\operatorname{ad} \lambda)(X) \quad \forall X \in \mathcal{H}, \quad r^{*}(\lambda)(Y)=\frac{1}{2} \operatorname{coth}\left(\frac{1}{2} \operatorname{ad} \lambda\right)(Y) \quad \forall Y \in \mathcal{H}^{\perp} .
$$

We here use the Laurent series expansion of $\frac{1}{2} \operatorname{coth}\left(\frac{z}{2}\right)$ around $z=0$, the $z^{-1}$ term in the expansion corresponds to the operator $(\operatorname{ad} \lambda)^{-1}$ on $\mathcal{H}^{\perp}$. The open domain $\check{\mathcal{H}} \subset \mathcal{H}$ is restricted by the condition ${ }^{2}$ that $r^{*}(\lambda)$ must be well defined by formula $\left(\overline{3} \overline{3} . \bar{s}^{\prime}\right)$ for $\lambda \in \check{\mathcal{H}}$. By using the identification $\operatorname{End}(\mathcal{G}) \simeq \mathcal{G} \otimes \mathcal{G}$ defined by the scalar product on $\mathcal{G}$, it can be shown that $r^{*}$ solves the CDYBE on $\mathcal{H} \subset \mathcal{G}$ :

$$
\left[r_{12}^{*}(\lambda), r_{23}^{*}(\lambda)\right]+H_{1}^{i} \frac{\partial}{\partial \lambda^{i}} r_{23}^{*}(\lambda)+\text { cycl. perm. }=-\frac{1}{4} \hat{f}, \quad \lambda \in \check{\mathcal{H}} \subset \mathcal{H},
$$

where $H^{i}$ denotes a basis of $\mathcal{H}$.

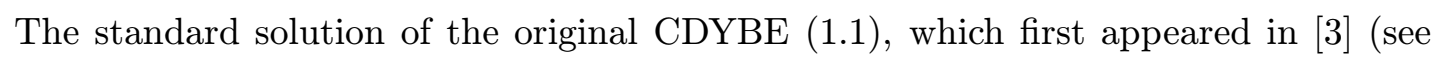

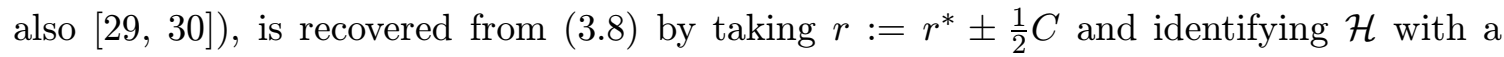
Cartan subalgebra of a simple Lie algebra.

The passage from $r^{0}$ to $r^{*}$ corresponds to Dirac reduction in two senses. First, the phase space $\mathcal{M}_{\breve{G}}$ equipped with the canonical exchange $r$-matrix can be reduced by restricting the monodromy to $\exp (\check{\mathcal{H}})$, whereby the Dirac brackets of the chiral WZNW field take the same form as the PB in (2.2.

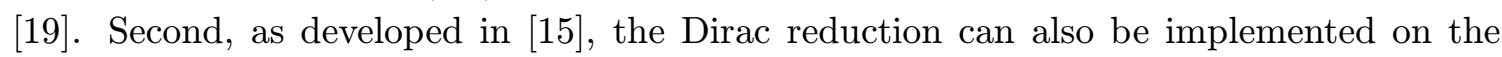
Poisson-Lie groupoids that enter the geometric interpretation of the CDYBE introduced in [i]

Finally, it is worth noting that formula (3.8) contains Felder's celebrated spectral parameter dependent elliptic dynamical $r$-matrices [4i $\left[\begin{array}{l}4 \\ 0\end{array}\right]$ and some generalizations of them,

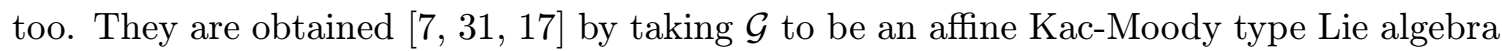
with $\mathcal{H}$ being a grade zero subalgebra in an integral gradation, and applying an evaluation homomorphism.

\section{Exchange $r$-matrix compatible with any PL structure}

We have seen that by an appropriate choice of the exchange $r$-matrix the gauge action $(\overline{3} \cdot \overline{3})$ can be interpreted as a classical $G$-symmetry on $\mathcal{M}_{\breve{G}}$. Interestingly, we can achieve the same with respect to any (coboundary) Poisson-Lie (PL) structure on $G$.

\footnotetext{
${ }^{2} \mathrm{~A}$ non-empty domain exists, for example, if $\mathcal{H}$ is a reductive subalgebra of a simple Lie algebra.
} 
Let us equip the group $G=\{h\}$ with a PL structure by means of the Sklyanin bracket

$$
\kappa\{h \stackrel{\otimes}{,} h\}_{R^{\nu}}=\left[h \otimes h, R^{\nu}\right],
$$

where $R^{\nu} \in \mathcal{G} \wedge \mathcal{G}$ is a constant $r$-matrix satisfying

$$
\left[R_{12}^{\nu}, R_{23}^{\nu}\right]+\text { cycl. perm. }=-\nu^{2} \hat{f}
$$

for some constant $\nu$. (Note that $\nu$ must be purely imaginary, or zero, if $G$ is compact.) Then look for the conditions on $r(M)$ that guarantee the standard right action ( $\left(\overline{3}, \bar{l}_{1}\right)$ of $G$ on $\mathcal{M}_{\breve{G}}$ to be a PL action. In fact, we find the requirement

$$
K\left(h M h^{-1}\right)=(h \otimes h) K(M)\left(h^{-1} \otimes h^{-1}\right) \quad \text { for } \quad K(M):=r(M)-R^{\nu} .
$$

This means that the gauge action ( $(\overline{3} \cdot \overline{1})$ of $\left(G,\{,\}_{R^{\nu}}\right)$ on $\left(\mathcal{M}_{\breve{G}},\{,\}_{W Z}^{r}\right)$ is a PL symmetry if and only if the exchange r-matrix $r(M)$ is such a solution of (2.6) for which the difference $\left(r(M)-R^{\nu}\right)$ is G-equivariant.

By using the substitution $r(M)=R^{\nu}+K(M)$ together with (4.2') and the equivariance

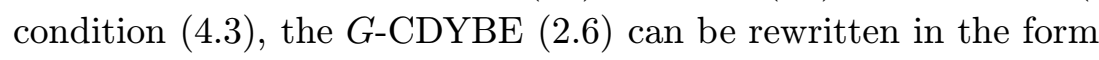

$$
\left[K_{12}(M), K_{23}(M)\right]-\frac{1}{2} T_{1}^{a} \mathcal{D}_{a}^{+} K_{23}(M)+\text { cycl. perm. }=\left(\frac{1}{4}-\nu^{2}\right) \hat{f} .
$$

This equation for an equivariant $K: \check{G} \rightarrow \mathcal{G} \wedge \mathcal{G}$ may be referred to as the $P L-C D Y B E$ on $G$ since it guarantees PL $G$-symmetry on the chiral WZNW phase space. It is remarkable that the reference $r$-matrix $R^{\nu}$ enters into this equation only through the constant $\nu$ in $(\overline{4} .2)$.

In a neighbourhood of $e \in G$, it is natural to search for $K(M)$ with the aid of the ansatz

$$
K(M)=\left\langle T_{a}, f_{\nu}(\operatorname{ad} m) T_{b}\right\rangle T^{a} \otimes T^{b}, \quad m=\log M,
$$

where $f_{\nu}(z)$ is assumed to be a holomorphic, odd complex function in a neighbourhood of

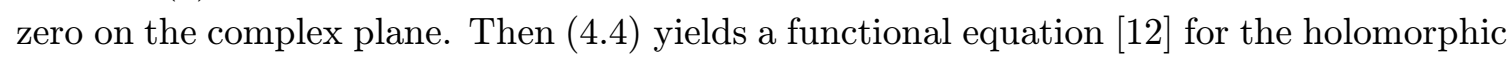
function $f_{\nu}$, whose unique solution is found to be

$$
f_{\nu}(z)=\frac{1}{2} \operatorname{coth} \frac{z}{2}-\nu \operatorname{coth} \nu z .
$$

The exchange $r$-matrices provided by this result were first found in $[\overline{8}] \overline{1}$ by using a different (more complicated) method, their uniqueness under the ansatz (4) has been established in [i] $[1 \overline{2} \overline{2}]$.

Some further remarks are here in order. First, note that for $\nu=0 f_{\nu}$ in (4.6) becomes the function $f_{0}$ in $\left(\bar{p}^{3} \cdot \bar{b}_{i}\right)$. The corresponding exchange $r$-matrix is thus compatible with classical $G$-symmetry (for $R^{0}=0$ ) as well as with PL symmetry for any antisymmetric solution $R^{0} \neq 0$ of the CYBE. Second, if $\nu=\frac{1}{2}$ then $r=R^{\frac{1}{2}}$, which is the case of the constant exchange $r$-matrices. Third, as mentioned before, for a compact Lie algebra $\mathcal{G}$ constant exchange $r$-matrices do not exist, because of the negative sign on the right-hand

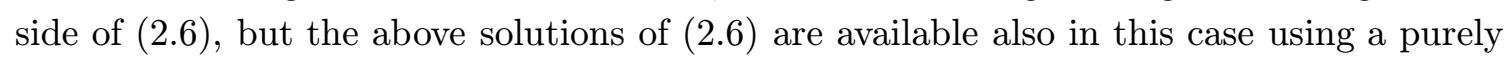
imaginary $\nu$ in (4). 


\section{Finite dimensional phase spaces related to WZNW}

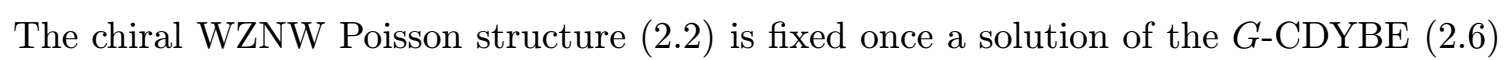
is given. It appears interesting that the WZNW exchange $r$-matrices also encode the PBs on certain finite dimensional Poisson manifolds. Indeed, it has been found in [i్n, that on the manifold

$$
P:=\check{G} \times G \times \check{G}=\left\{\left(M^{L}, g, M^{R}\right)\right\},
$$

the following formula defines a $P B,\{,\}_{P}^{r}$, for any solution $r: \check{G} \rightarrow \mathcal{G} \wedge \mathcal{G}$ of the $G$-CDYBE:

$$
\begin{aligned}
& \kappa\left\{g_{1}, g_{2}\right\}_{P}^{r}=g_{1} g_{2} r\left(M^{R}\right)-r\left(M^{L}\right) g_{1} g_{2} \\
& \kappa\left\{g_{1}, M_{2}^{R}\right\}_{P}^{r}=g_{1} M_{2}^{R} \Theta\left(M^{R}\right) \\
& \kappa\left\{g_{1}, M_{2}^{L}\right\}_{P}^{r}=M_{2}^{L} \Theta\left(M^{L}\right) g_{1} \\
& \kappa\left\{M_{1}^{R}, M_{2}^{R}\right\}_{P}^{r}=M_{1}^{R} M_{2}^{R} \Delta\left(M^{R}\right) \\
& \kappa\left\{M_{1}^{L}, M_{2}^{L}\right\}_{P}^{r}=-M_{1}^{L} M_{2}^{L} \Delta\left(M^{L}\right) \\
& \kappa\left\{M_{1}^{R}, M_{2}^{L}\right\}_{P}^{r}=0 .
\end{aligned}
$$

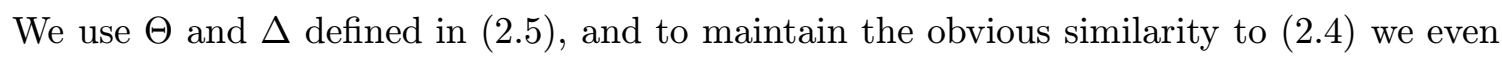
included the arbitrary constant $\kappa$ (the classical level parameter) into this definition. We stress that $g \in G$ is here $x$-independent. In fact, $\left(P,\{,\}_{P}^{r}\right)$ is an example of a Poisson-Lie groupoid in the sense of [i32]. This PL groupoid 'extracted' from $\left(\mathcal{M}_{\breve{G}},\{,\}_{W Z}^{r}\right)$ provides a geometric interpretation of the $G$-CDYBE analogous to the interpretation of the Etingof-

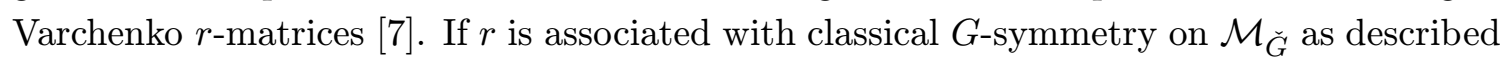
in Section 3, then our PL groupoid is essentially identical with the 'dynamical PL groupoid'

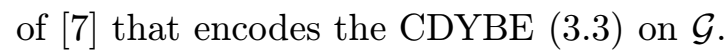

In analogy with the CDYBE on $\mathcal{G}$, the PL-CDYBE (14.4') admits a 'canonical' interpretation [i] $[\overline{2}]$ in terms of well known objects of PL geometry, which is nicer than the general case $(5.5)$. To describe this, let us now denote the elements of $P$ differently as

$$
P:=\check{G} \times G \times \check{G}=\left\{\left(\Omega^{L}, g, \Omega^{R}\right) \mid \Omega^{L, R} \in \check{G}, g \in G\right\},
$$

where $\check{G} \subset G$ is some open submanifold. Then let $\mathcal{R}:=R^{\frac{1}{2}} \in \mathcal{G} \wedge \mathcal{G}$ be a constant solution

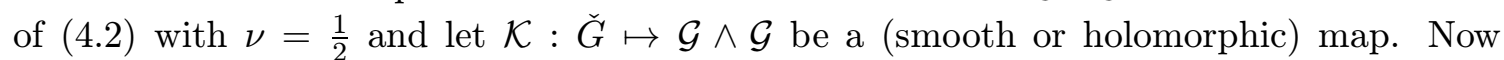
consider the following ansatz for a $\mathrm{PB},\{,\}_{c a n}$, on $P$ :

$$
\begin{aligned}
& \left\{g_{1}, g_{2}\right\}_{\text {can }}=\left(\mathcal{R}+\mathcal{K}\left(\Omega^{L}\right)\right) g_{1} g_{2}-g_{1} g_{2}\left(\mathcal{R}+\mathcal{K}\left(\Omega^{R}\right)\right) \\
& \left\{g_{1}, \Omega_{2}^{R}\right\}_{\text {can }}=g_{1}\left(\mathcal{R}^{-} \Omega_{2}^{R}-\Omega_{2}^{R} \mathcal{R}^{+}\right) \\
& \left\{g_{1}, \Omega_{2}^{L}\right\}_{\text {can }}=\left(\mathcal{R}^{-} \Omega_{2}^{L}-\Omega_{2}^{L} \mathcal{R}^{+}\right) g_{1} \\
& \left\{\Omega_{1}^{R}, \Omega_{2}^{R}\right\}_{\text {can }}=-\mathcal{R} \Omega_{1}^{R} \Omega_{2}^{R}-\Omega_{1}^{R} \Omega_{2}^{R} \mathcal{R}+\Omega_{1}^{R} \mathcal{R}^{-} \Omega_{2}^{R}+\Omega_{2}^{R} \mathcal{R}^{+} \Omega_{1}^{R} \\
& \left\{\Omega_{1}^{L}, \Omega_{2}^{L}\right\}_{\text {can }}=\mathcal{R} \Omega_{1}^{L} \Omega_{2}^{L}+\Omega_{1}^{L} \Omega_{2}^{L} \mathcal{R}-\Omega_{1}^{L} \mathcal{R}^{-} \Omega_{2}^{L}-\Omega_{2}^{L} \mathcal{R}^{+} \Omega_{1}^{L} \\
& \left\{\Omega_{1}^{R}, \Omega_{2}^{L}\right\}_{\text {can }}=0 .
\end{aligned}
$$

Note that $\mathcal{R}^{ \pm}:=\mathcal{R} \pm \frac{1}{2} C$ and that the dynamical $r$-matrix $\mathcal{K}$ appears only in the first line of formula $\left({ }_{0} \cdot 4\right)$. We assume that $\mathcal{K}$ is a $G$-equivariant map, since anyhow this is required 
locally around $e \in G$ by the Jacobi identity $\left\{\left\{g_{1}, g_{2}\right\}_{\text {can }}, \Omega_{3}^{L}\right\}_{\text {can }}+$ cycl. perm $=0$ and its counterpart with $\Omega^{R}$. The only nontrivial Jacobi identity to check is the one involving $\left\{\left\{g_{1}, g_{2}\right\}_{c a n}, g_{3}\right\}_{c a n}$. This condition is found to be equivalent to the following version of the $P L-C D Y B E$ :

$$
\left[\mathcal{K}_{12}, \mathcal{K}_{23}\right]+\frac{1}{2} T_{1}^{a} \mathcal{D}_{a}^{+} \mathcal{K}_{23}+\text { cycl. perm. }=\mathcal{I} \quad \text { on } \quad \check{G}
$$

where $\mathcal{I}$ is an arbitrary $G$-invariant constant element of $\mathcal{G} \wedge \mathcal{G} \wedge \mathcal{G}$.

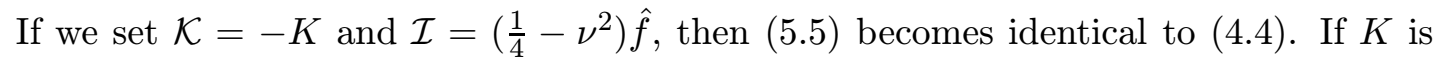

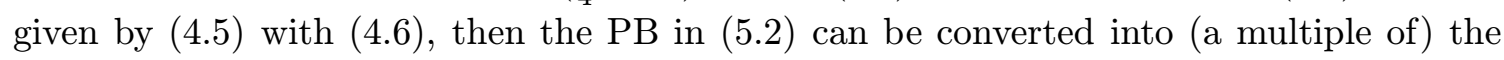
$\mathrm{PB}$ in $(5.4)$ by a certain change of variables. This will be described in detail in a future publication.

In fact [333, $3 \overline{3} \overline{4}]$, for $\mathcal{K}=0$ the PB $(\overline{5}-4)$ becomes the canonical PB of the Heisenberg double of the PL group $G$ equipped with the Sklyanin PB that belongs to $\mathcal{R}$, if one further sets $\Omega^{R}=g^{-1} \Omega^{L} g$. Thus $\Omega^{L}$ and $\Omega^{R}$ define directly the momentum maps that generate the natural PL actions of $G$ on $\left(P,\{,\}_{\text {can }}\right)$ that act by left and right-multiplications on $g$.

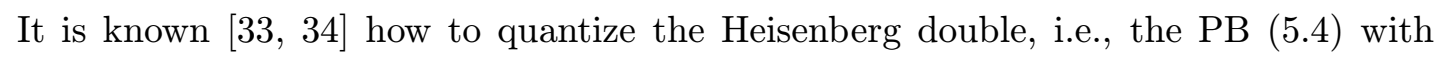
$\mathcal{K}=0$. It is an interesting open problem to perform the quantization of $\left(P,\{,\}_{\text {can }}\right)$ in the case

$$
\mathcal{K}(\Omega)=-\left\langle T_{a}, f_{\nu}(\operatorname{ad}(\log \Omega)) T_{b}\right\rangle T^{a} \otimes T^{b}
$$

with the function $f_{\nu}$ in $(\overline{4}-6)$, which are the unique solutions of the PL-CDYBE ( in terms of a complex analytic function. One should consider the quantization of the PB $(\overline{5} .2)$ also in the general case; the resulting structure should be related to the 'quantum algebraic properties' of the WZNW conformal field theory. This appears to be a natural idea in the context of the programme to canonically quantize the WZNW model and to

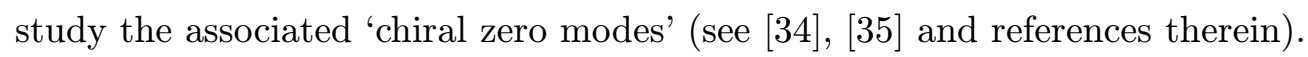

\section{Concluding remarks}

The results reported so far in this talk can be extended in several directions. For example, one can consider the dynamical $r$-matrices that arise in the classical WZNW model with twisted boundary condition associated with a finite order automorphism, $\mu$, of the group $G$. Denote also by $\mu$ the induced automorphism of $\mathcal{G}$, of order $N$ say, and suppose that it preserves the 'scalar product' $\langle$,$\rangle on \mathcal{G}$. In this case the full WZNW field satisfies $g_{W Z}(\sigma+2 \pi, \tau)=\mu\left(g_{W Z}(\sigma, \tau)\right)$ and the corresponding chiral fields obey

$$
g(x+2 \pi)=\mu(g(x)) M, \quad M \in G .
$$

Let us assume for simplicity that $G$ is a complex simple Lie group, and use the decomposition

$$
\mathcal{G}=\oplus_{a=0}^{N-1} \mathcal{G}_{a}, \quad \mu(X)=\exp \left(2 \pi i \frac{a}{N}\right) X \quad \forall X \in \mathcal{G}_{a} .
$$

In order to obtain a twisted analogue of the canonical $r$-matrix, we restrict the monodromy to the form $M=e^{\lambda}$ with $\lambda$ varying in an open domain, $\check{\mathcal{G}}_{0}$, in $\mathcal{G}_{0}$. Then we enquire about 
the PB of the $\mu$-twisted chiral WZNW field under the assumption that it enjoys classical $G_{0}$-symmetry. In fact, we find that such a $\mathrm{PB}$ again has the form $\left(\begin{array}{l}2 \\ 2\end{array} 2 \frac{1}{2}\right)$ with the exchange $r$-matrix $r_{\mu}: \check{\mathcal{G}}_{0} \rightarrow \mathcal{G} \otimes \mathcal{G} \simeq \operatorname{End}(\mathcal{G})$ given by

$$
r_{\mu}(\lambda)=\left\{\begin{array}{cc}
f_{0}(\operatorname{ad} \lambda) & \text { on } \mathcal{G}_{0} \\
\frac{1}{2} \operatorname{coth}\left(\frac{1}{2} \operatorname{ad} \lambda-i \pi \frac{a}{N}\right) & \text { on } \mathcal{G}_{a} \text { for } a \neq 0,
\end{array}\right.
$$

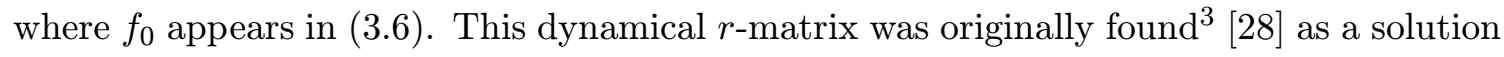
of the CDYBE on $\mathcal{G}_{0}$ (eq. $\left(\overline{3}_{2} .9_{i}\right)$ with $\left.\mathcal{H}:=\mathcal{G}_{0}\right)$. It can be derived by calculating the PBs on the $\mu$-twisted chiral WZNW phase space with $M \in \check{G}_{0}$ and the symplectic form defined by using $\left(\overline{2} . \overline{2}_{3}^{\prime}\right)$ with the restriction of $\rho_{0}$ in $\left(\overline{3} \overline{3}_{-} \bar{Z}_{i}\right)$ to $\check{G}_{0}$. If $\mathcal{G}_{0}$ is non-Abelian, then further Dirac reduction to a Cartan subalgebra of $\mathcal{G}_{0}$ (and intermediate cases) is also possible, yielding 'twisted analogues' of the $r$-matrices in (3. Wakimoto type free field realizations of the chiral WZNW field can be worked out following the lines of [i] 13 in].

So far I have performed the above analysis under the simplifying assumption that $\mathcal{G}$ is complex and simple, but it should not be difficult to generalize it to any self-dual Lie algebra equipped with a finite order automorphism compatible with the scalar product.

Dynamical exchange $r$-matrices appear not only in the (twisted) chiral WZNW model, but also in its intriguing generalization introduced recently by Klimcik. In particular, Felder's $r$-matrix [4] natural to expect that this model with twisted boundary condition should accommodate

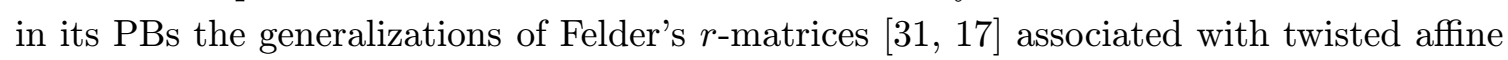
Kac-Moody algebras, but further work is needed to clarify the situation.

Another problem, which is currently under investigation, concerns the correspondence between some of the $r$-matrices mentioned in the talk and spin Calogero-Moser type integrable systems. At the classical level, it seems straightforward how to generalize the

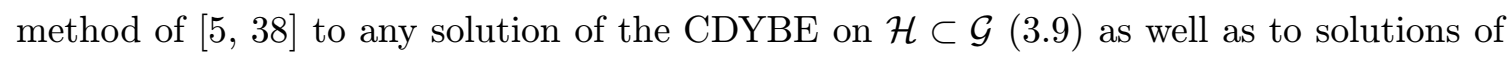
the spectral parameter dependent CDYBE on self-dual Lie algebras. It is not clear however if this method will lead to new and interesting integrable systems or not. We also would like to see if the natural generalization of the CDYBE on $\mathcal{G}$ given by the PL-CDYBE (5.5.5. is related to (perhaps spin Ruijsenaars type) integrable systems. I hope to report on these questions on another occasion.

\section{Acknowledgments}

I wish to thank the organizers of the meeting at IFT for the invitation to Sao Paulo and for the superb hospitality. This work was also supported by the Hungarian Scientific Research Fund (OTKA) under T034170, T029802, T030099, M028418 and M036804.

\footnotetext{
${ }^{3}$ In [를
} 


\section{References}

[1] J.-L. Gervais and A. Neveu, iNucl. Phys. B 238 11984$) 125$

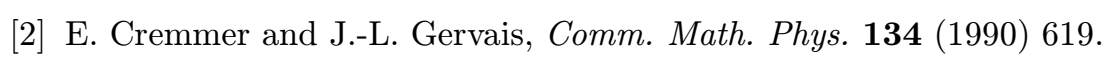

[3] J. Balog, L. Dạbrowski and L. Fehér, 'P

[4] G. Felder, pp. 1247-1255 in: Proc. ICM Zürich, 1994 (Birkhäuser, 1994), 'hepep-th/940715

[5] J. Avan, O. Babelon and E. Billey, Comm. Math. Phys. 178 (1996) 281, hep-th/9505091;

[6] P. Etingof and O. Schiffmann, Lectures on the dynamical Yang-Baxter equations, math QQA/9908064.

[7] P. Etingof and A. Varchenko, 'Comm. Math. Phys. 192 (1998) 777, 'q-aig/9703040!

[8] J. Balog, L. Fehér and L. Palla, Phys. Lett.B $\mathbf{4 6 3}$ (1999) 83, hep-th/9907050;

J. Balog, L. Fehér and L. Palla, Nucl. Phys. B 568 (2000) 503, hep-th/9910046.

[9] K. Gawędzki, ¿'Comm. Math. Phys. 139 $199 \overline{1}) 2011$.

[10] F. Falceto and K. Gawędzki, 'J. Geom. Phys. 11 (1993) 251 i hep-th/9209076.

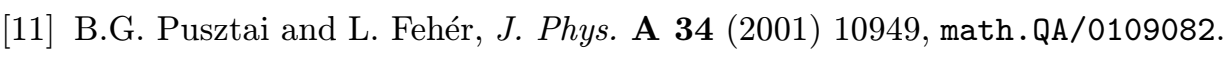

[12] L. Fehér and I. Marshall, On a Poisson-Lie analogue of the classical dynamical Yang-Baxter equation for self-dual Lie algebras, math.QA/0208159.

[13] J. Balog, L. Fehér and L. Palla, Ju. Phys. A 33 (2000) 945ín hep-th/9910112.

[14] J. Balog, L. Fehér and L. Palla, iPhys. Lett. A 277 (2000) 107', hepeth/0007045!

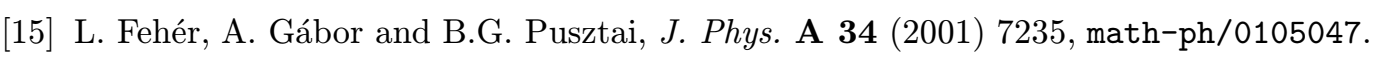

[16] L. Fehér and A. Gábor, pp. 331-336 in: Quantum Theory and Symmetries, eds. E. Kapuscik et al (World Scientific, 2002), hep-th/0111252

[17] L. Fehér and B.G. Pusztai, Núcl. Phys. B 621_(2002) 622, math.QA/0109132i.

[18] J. Balog, L. Fehér and L. Palla, pp. 1-19 in: CRM Proceedings and Lectures Notes, Volume 26, eds. J. Harnad et al (AMS, 2000), hep-th/9912173.

[19] L. Fehér, 'Phys. Atom. Nucl. 65_(2002) 1023', math-ph/0104027.

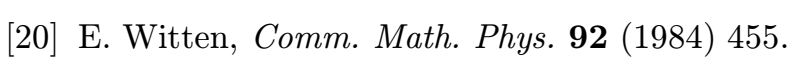

[21] A. Alekseev, Y. Kosmann-Schwarzbach and E. Meinrenken, 'Can. math.DG/0006168.

[22] O. Babelon, 'P

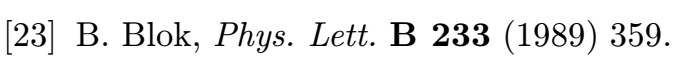

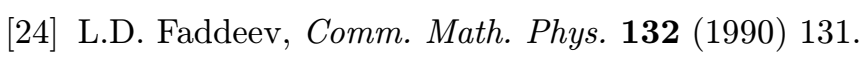

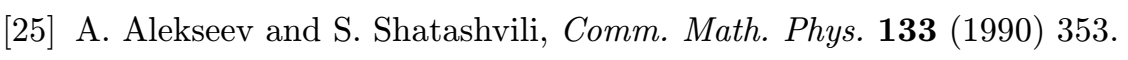

[26] M. Cahen, S. Gutt and J. Rawnsley, 'Contemp. Math. 179.1994) 1.'.

[27] A. Alekseev and E. Meinrenken, invent. Math. 139 $(2000) 1351$ math.DG/9903052.

[28] P. Etingof and O. Schiffmann, Math. Res. Lett. 8 (2001) 157, math.QA 0005282 


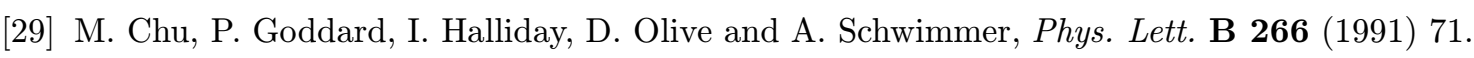

[30] O. Babelon, F. Toppan and L. Bonora, 'Comm. Math. Phys. 140 (1991) 933.

[31] P. Etingof and O. Schiffmann, Math. Res. Lett. 6. (1999) 593 , math.QA $9908115 !$

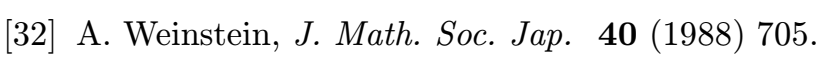

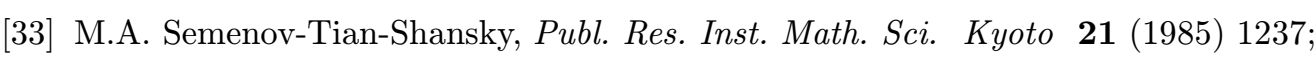
M.A. Semenov-Tian-Shansky, Theor. Math. Phys. 93 (1992) 1292, hep-th/9304042,

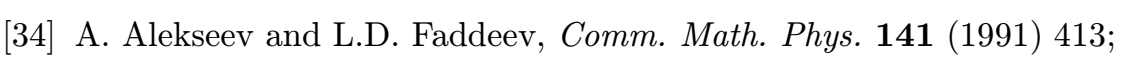

A. Alekseev and L.D. Faddeev, An involution and dynamics for the $q$-deformed quantum top, hep-th/9406196.

[35] P. Furlan, L.K. Hadjiivanov and I.T. Todorov, Chiral zero modes of the SU(n) WZNW model, hep-th/02 11154

[36] A. Alekseev and E. Meinrenken, Clifford algebras and the classical dynamical Yang-Baxter equation, math.RT/0209347i:

[37] C. Klimcik, Quasitriangular WZW model, 'hep-th/0103118'.

[38] L.C. Li and P. Xu, 'Comm. Math. Physs. 231 (2002) 257, math.QA/0105162i 